\begin{tabular}{|c|c|}
\hline \multirow{3}{*}{ 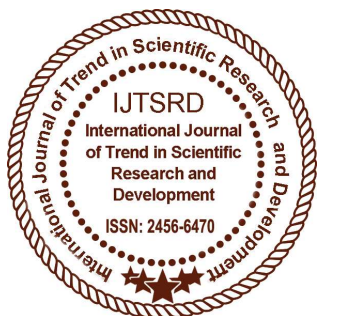 } & $\begin{array}{l}\text { International Journal of Trend in Scientific } \\
\text { Research and Development (IJTSRD) }\end{array}$ \\
\hline & International Open Access Journal \\
\hline & ISSN No: 2456 - 6470 | www.ijtsrd.com | Volume - 2 | Issue - 2 \\
\hline
\end{tabular}

\title{
Basics and Features of Artificial Neural Networks
}

\author{
Rajesh CVS \\ Assistant Professor \\ Department of Mechanical Engineering, \\ Avanthi Institute of Engineering \& Technology, \\ Vizianagaram, Andhra Pradesh, India
}

\author{
M. Padmanabham \\ Assistant Professor \\ Department of Mechanical Engineering, \\ Viswanadha Institute of Technology \& \\ Management, Visakhapatnam, Andhra Pradesh, India
}

\section{ABSTRACT}

The models of the computing for the perform the pattern recognition methods by the performance and the structure of the biological neural network. A network consists of computing units which can display the features of the biological network. In this paper, the features of the neural network that motivate the study of the neural computing are discussed and the differences in processing by the brain and a computer presented, historical development of neural network principle, artificial neural network (ANN) terminology, neuron models and topology $S$ are discussed.

Keywords: Biological Neural Networks, Terminology in Artificial Neural Networks, Models of Neuron and Topology

\section{INTRODUCTION:}

In the neural network characteristics, few of the attractive features of biological neural network make superior to the most complicated in Artificial Intelligent recognition tasks.

Biological Neural Networks: The basic unit of the network is known as a neuron or a nerve cell. Mainly it consists of a cell body or soma in which the cell nucleus is located. Nerve fibers like tree form called as dendrites and these are associated with the cell body. Dendrites are receiving the signals from the other neurons.

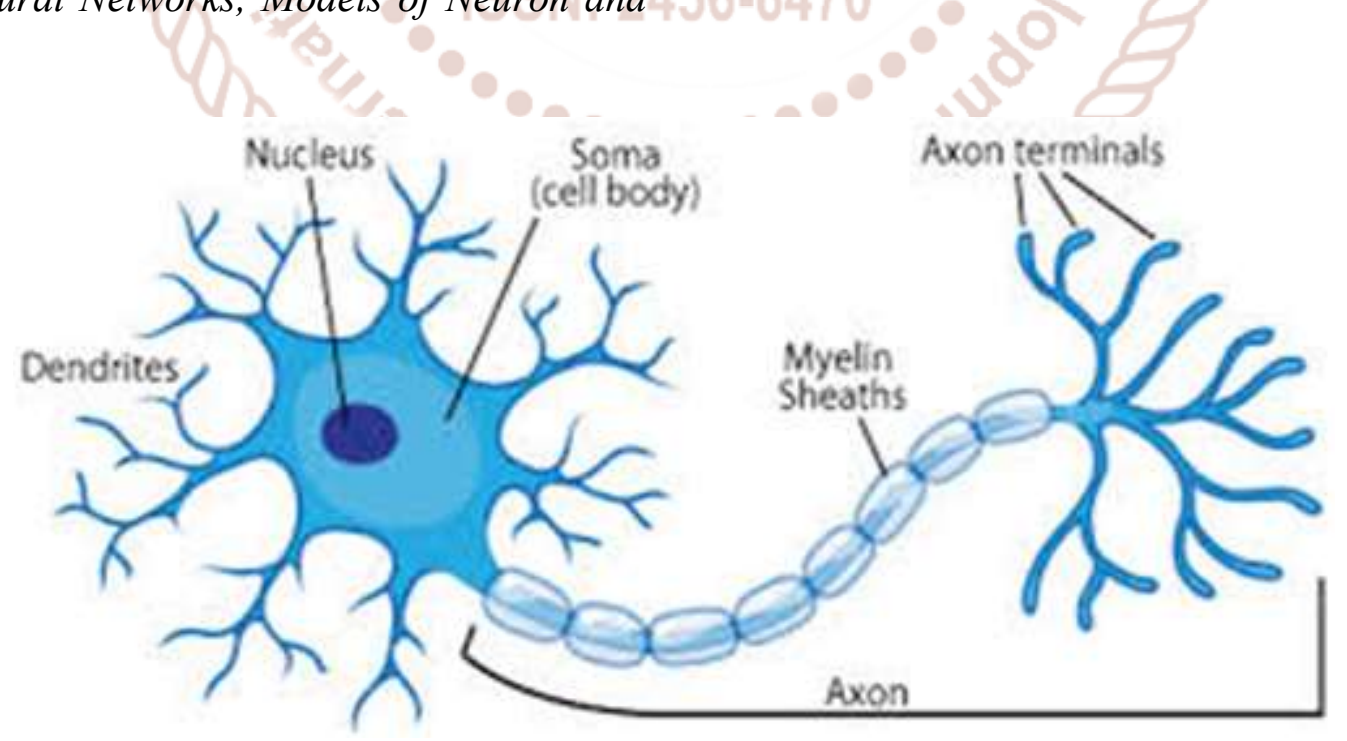




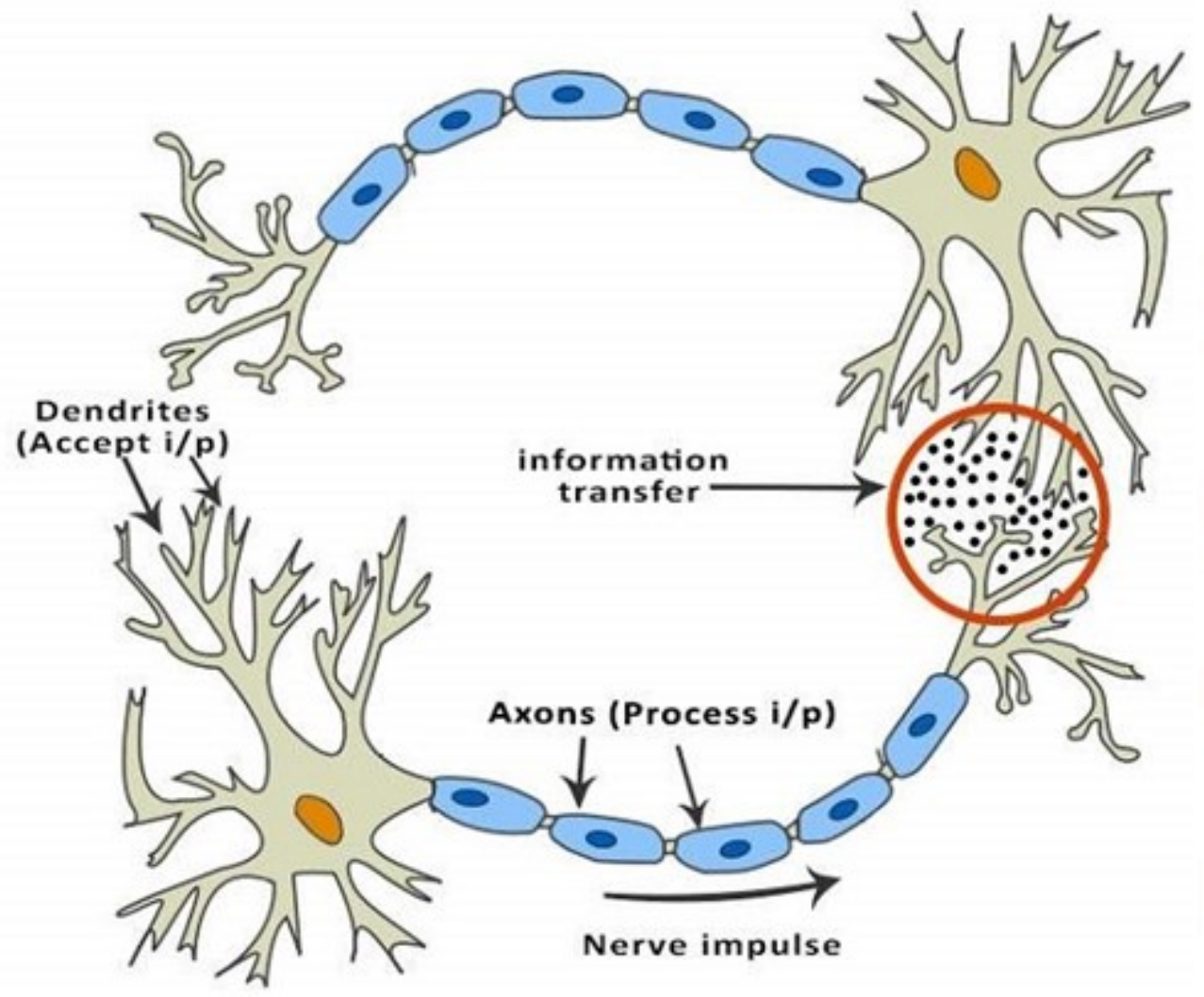

The third type of neuron, which can receive the information from muscles or sensory organs like ear or eyes, is called as receptor neuron. A typical neuron cell body size is approximately 10-80 micrometers and the axons and dendrites have diameters of the order of a few micrometers.

\section{Performance Comparison of Computer and Biological Neural Networks:}

Neural Networks can perform massively parallel operations.

$>$ Neural Networks have the large number of computing elements.

$>$ The number of neurons in brain estimated about $10^{11}$ and the total interconnections are around $10^{15}$.
Neural Networks store information in the strengths of the interconnections. We know that, in computer information stored in the memory by its location.

In a computer, any new information in the same location may destroy the old information. But in Neural Network, new information added by the adjusting interconnection strengths without any destroying of old information.

Neural Networks are slow in processing information.

In Neural Networks, there is no central control for processing information in the brain.

In computer there is a control unit which controls the all activities. 


\section{Historical Development of Neural Network Principle:}

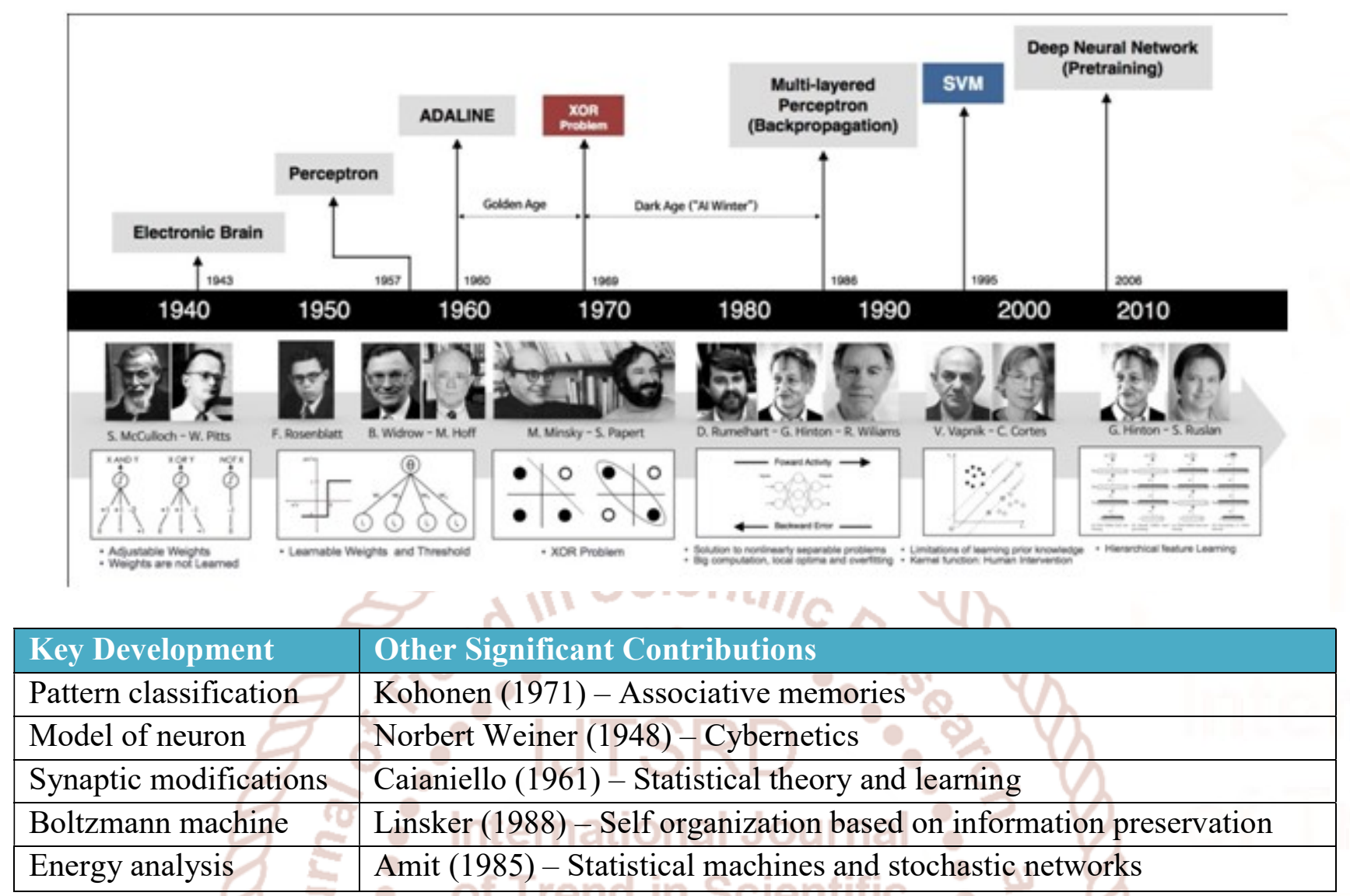

\section{Terminology in Artificial Neural Networks:}

Interconnections: According to the topology in an artificial neural network several processing units are interconnected to accomplish pattern recognition task.

Processing Unit: Artificial Neural Network (ANN) has highly simplified model of the structures of the biological neural network. ANN consists of interconnected processing units.

Operations: Each unit of an ANN receives inputs from other connected units or from an external source. The activation value determines the actual output from the output function unit.

\section{Models of Neuron:}

In this paper we consider the three classical models for an artificial neuron or processing unit.

\section{i) Mc Colloch-Pitts Model}

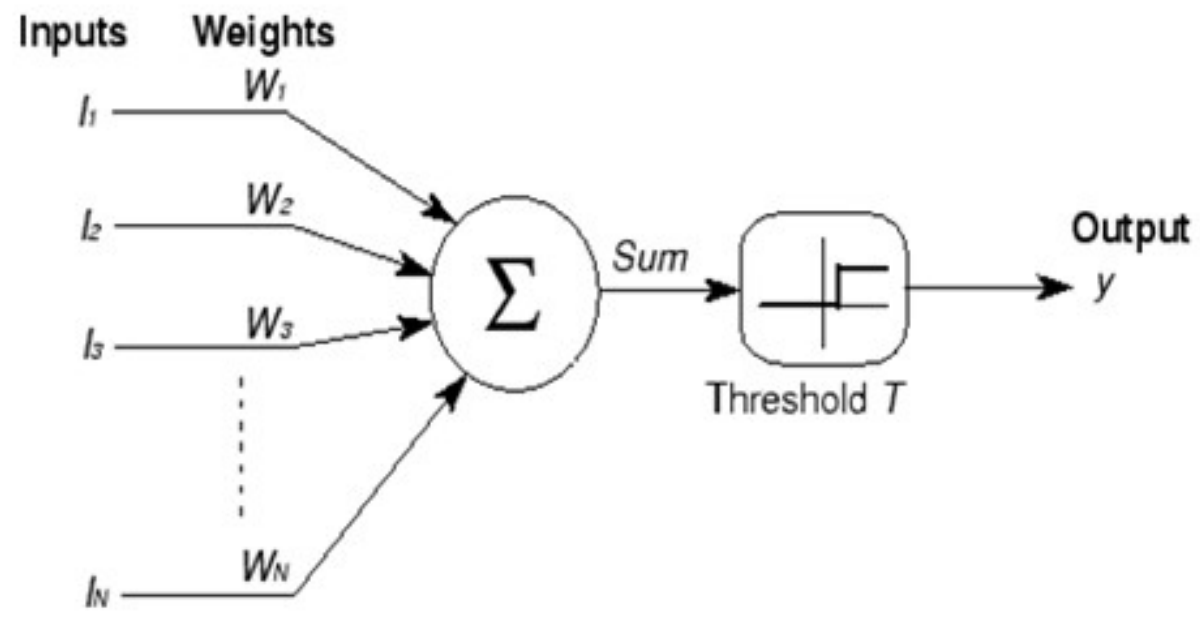




\section{ii) Perceptron}

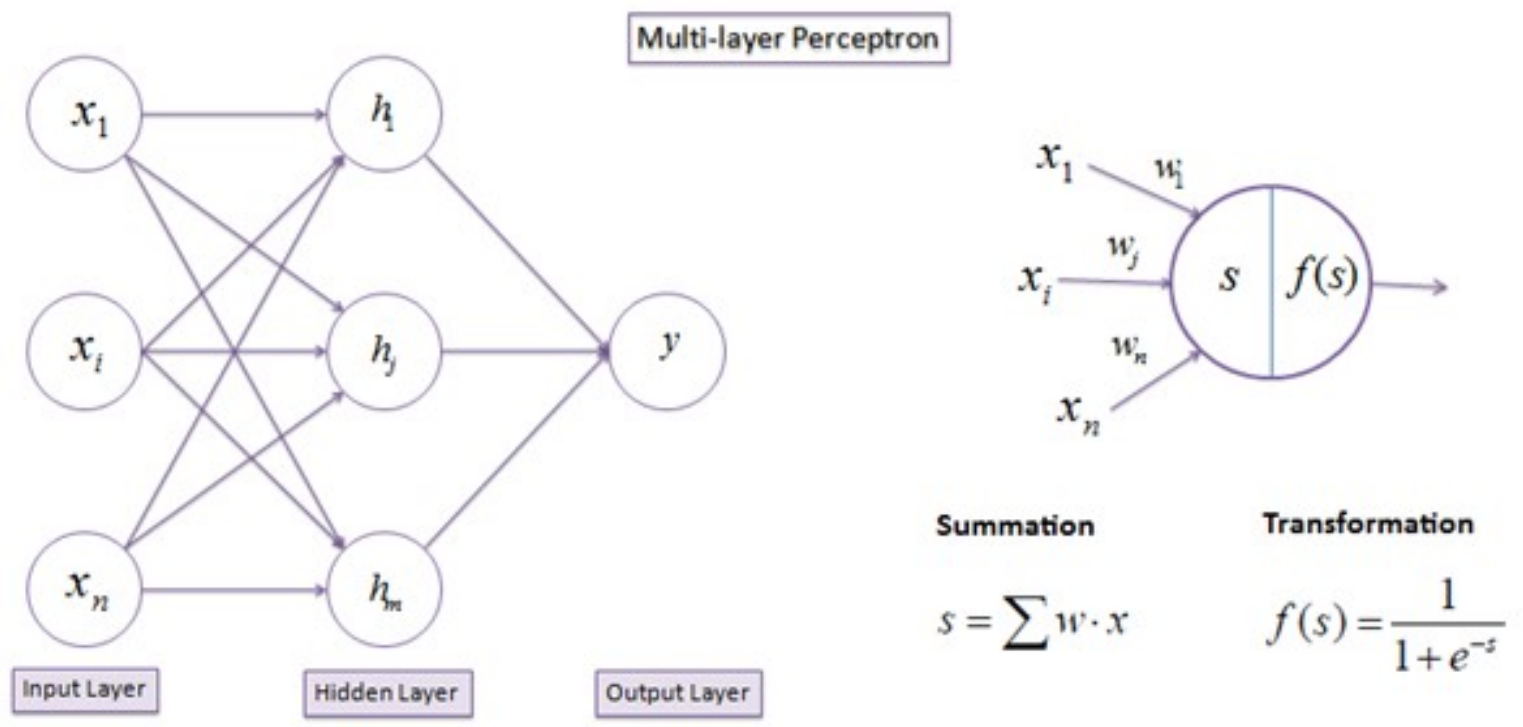

iii) Adaline

\section{ADALINE - ARCHITECTURE}

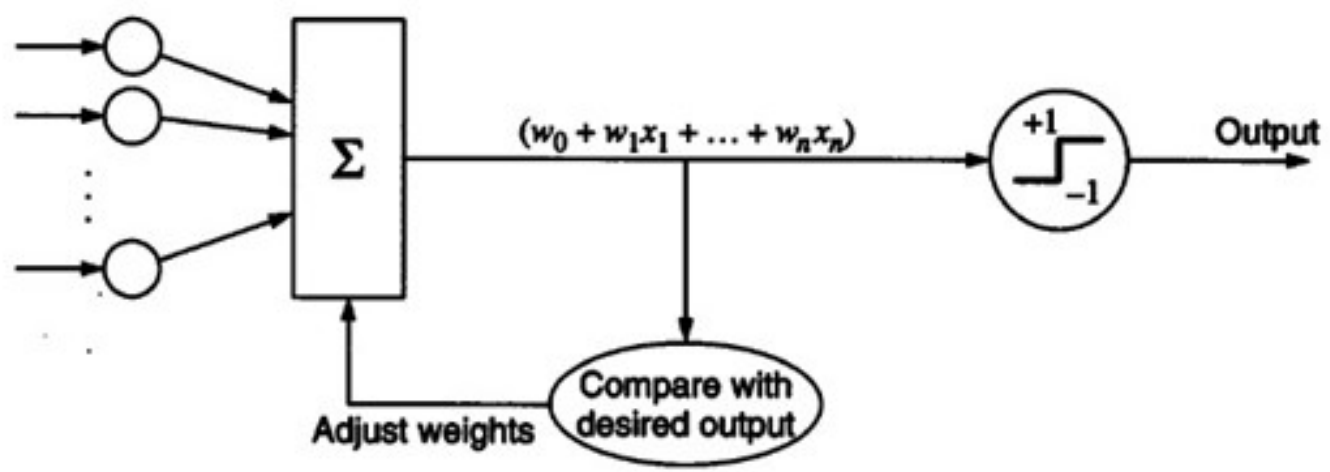

Topology: When the processing units are organized in a correct process to accomplish a given pattern recognition task, Artificial Neural Networks are useful.

The arrangement of the connections, patterns input/output and processing units are referred to topology.

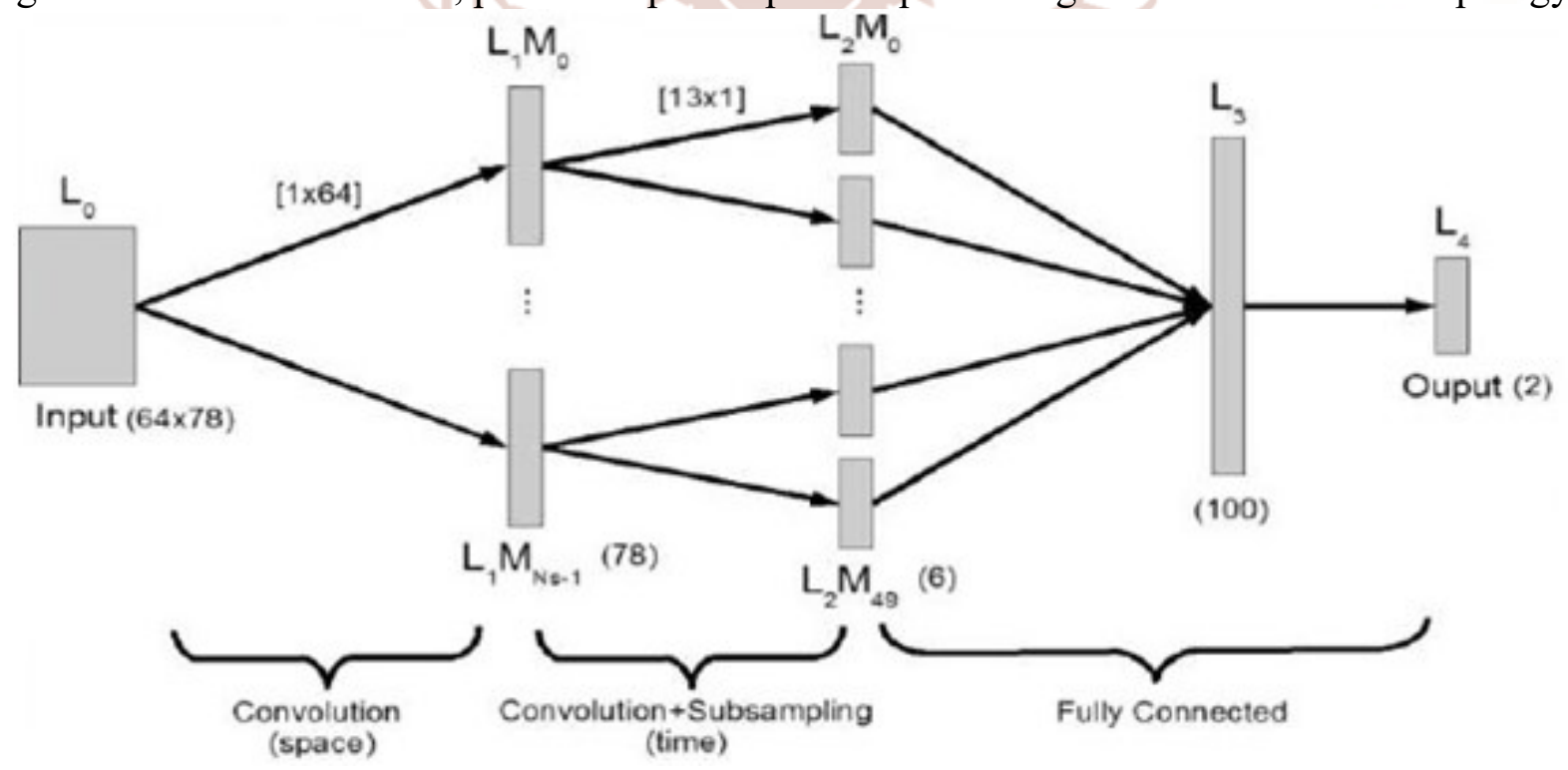




\section{Artificial Neural Network Topology}

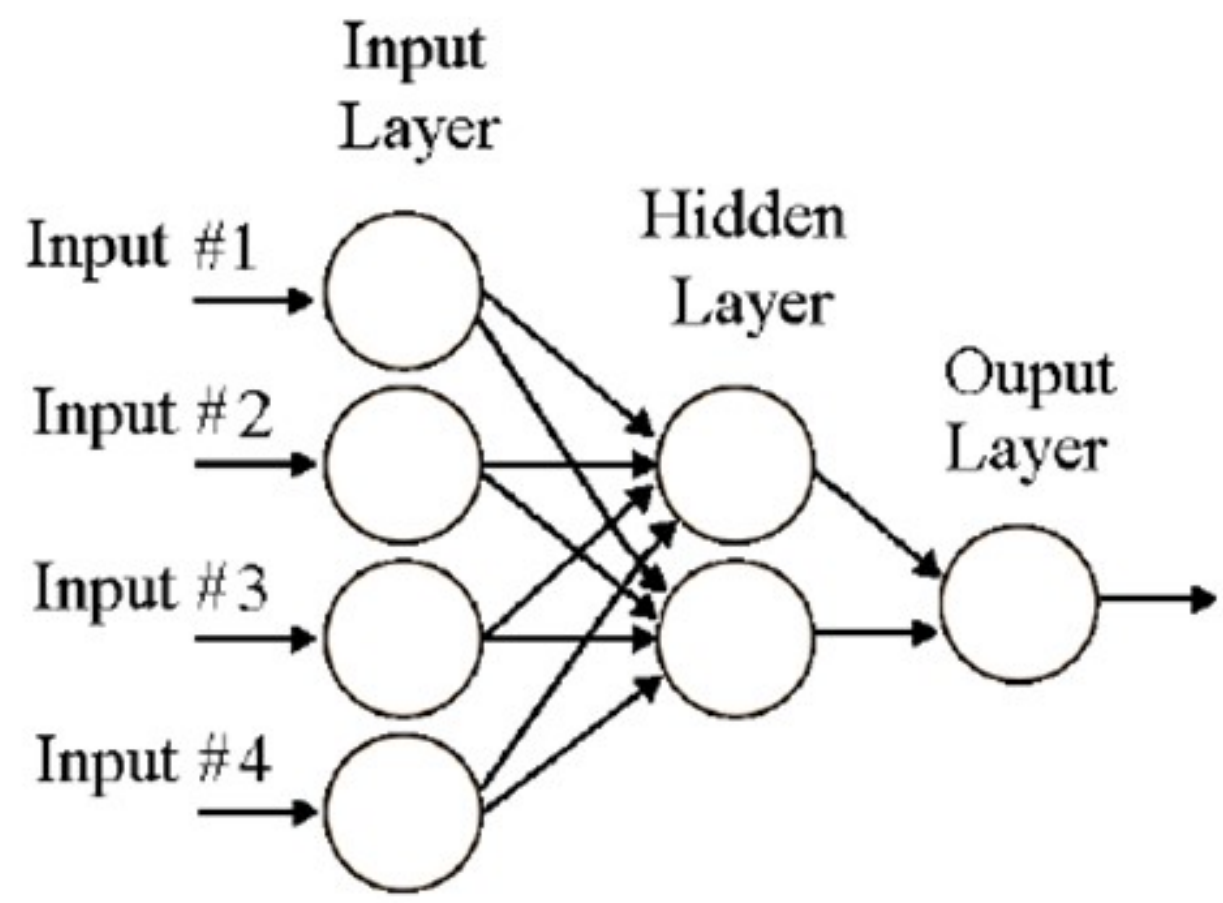

\section{Conclusion:}

This research paper will help you to understand about the biological neural networks, performance of computer and biological neural network, historical development of neural network principles, terminology in artificial neural network, models of neuron and topology.

\section{References:}

1. Gill, G. S. (2008), Election Result Forecasting Using Two Layer Perceptron Network, Journal of Theoretical and Applied Information Technology, 47(11),1019-1024

2. Caleiro, A. (2005), How to Classify a Government? Can a Neural Network do it? , University of Evora, Economics Working Papers.
3. Niska, H., Hiltunen, T., Karppinen, A., Ruuskanen, J., \& Kolehmainen, M. (2004), Evolving The Neural Network Model For Forecasting Air Pollution Time Series, Engineering Applications of Artificial Intelligence, 17(2), 159-167.

4. Gorunescu, F. (2011), Data Mining: Concepts, Models and Techniques (Vol. 12), Springerverlag Berlin Heidelberg.

5. Han, J., Kamber, M., \& Pei, J. (2006), Data Mining: Concepts And Techniques. Morgan Kaufmann.

6. Law, R., \& Au, N. (1999), A Neural Network Model To Forecast Japanese Demand For Travel To Hong Kong, Tourism Management, 20(1), 8997. 